\title{
Discusión Teórica Respecto A La Atención De Las Nee (Necesidades Educativas Especiales) En La Legislación Educativa Mexicana
}

\section{Theoretical Discussion Regarding The Care Of Nee (Special Needs) In Mexican Law Education.}

\author{
David Yoshio Rodríguez Mujica¹, Tania Márquez Feliciano², Mauricio Zacarías \\ Gutiérrez $^{3}$
}

1- Docente-investigador de la escuela Normal de Educación Primaria, Fray Matías de Córdova, Tapachula, Chiapas. Realizado en estos
momentos la investigación "Discurso de la formación docente, en estudiantes de Licenciatura en Educación Especial. Escuela normal Fray
Matías de Córdova. Tapachula, Chiapas" México. E-mail - mazag@hotmail.com 2- Licenciatura en Educación Especial. Realizan una indagación teórica respecto a la "Atención de las Necesidades Educativas Especiales (NEE) en la legislación educativa mexicana” 2sem. Escuela normal Fray Matías de Córdova. Tapachula, Chiapas” México.

3- Licenciatura en Educación Especial. Realizan una indagación teórica respecto a la "Atención de las Necesidades Educativas Especiales (NEE) en la legislación educativa mexicana” 2sem. Escuela normal Fray Matías de Córdova. Tapachula, Chiapas” México.

\section{Resumen}

El presente escrito tiene como objetivo explicar y dar a conocer parte de la legislación educativa mexicana enfocada a la educación especial y por ende a los alumnos que presentan NEE, con o sin discapacidad. Así mismo, relacionarlo con el proceso integración e inclusión en escuelas regulares. Para esto, es relevante tomar en cuenta algunos antecedentes normativos y el aporte de algunos teóricos. Tomar en cuenta las bases que conforman nuestra Política Educativa destinada a la integración y a la inclusión educativa, se vuelve puntos fundamentales. Por lo tanto, exige revisar detenidamente su aplicación a las escuelas que brinda atención a alumnos que presenten dificultades en su aprendizaje, mismas que pueden asociarse a una discapacidad; tomando en cuenta los objetivos que se plantean para que los alumnos logren su máximo desempeño escolar.

Palabras clave: Discusión, atención, NEE - Necesidades Educativas Especiales, legislación educativa mexicana.

42 MUJICA, D.Y.R.; FELICIANO, T.M.; GUTIÉRREZ, M.Z.: Discusión Teórica Respecto A La Atención De Las Nee (Necesidades Educativas Especiales) En La Legislación Educativa Mexicana. 


\begin{abstract}
The present paper aims to explain and publicize part of the Mexican educational legislation focused on special education and therefore students with SEN, with or without disabilities. Likewise, linking with the integration process and inclusion in regular schools. For this, it is important to consider some regulatory background and the contribution of some theorists. You take into account the bases that make up our education policy aimed at integration and educational inclusion, it becomes fundamental points. Therefore, carefully review your application requires schools to provide attention to students who bave difficulties in learning them can be associated with a disability; taking into account the objectives set for students to reach their full academic performance
\end{abstract}

Keywords: Discussion, attention, NEE - Special Educational Needs, Mexican educational legislation.

\section{Introducción}

Objetivos: Dar a conocer la atención de las necesidades educativas especiales desde la legislación educativa en México. Dar a conocer teóricamente como se ha atendido las NEE (necesidades educativas especiales) y los procesos de integración e inclusión de los alumnos que las presentan.

Nos enfocaremos en la definición de los conceptos: Discapacidad y Necesidades Educativas Especiales, ya que es relevante dejar claro estos dos términos utilizados para referirnos a las personas con alguna deficiencia y por ende entender más el trabajo en educación especial. Por otra parte, la descripción del proceso de Integración e Inclusión de alumnos con Nee en las escuelas regulares, se considera un tema de vital importancia, pues será aquí donde se defina cuáles son los pasos a llevar cabo para integrar e incluir a los alumnos con problemas de aprendizaje y de aquellos con alguna Nee, asociada a una Discapacidad.

Por último, en el tema de "Evaluación psicopedagógica en educación especial (trabajo en el aula)", se considera fundamental mencionar y describir la intervención docente en el trabajo con los alumnos con NEE, con o sin discapacidad. Explicando el proceso de evaluación y el seguimiento que se le da a los resultados con la propuesta curricular adaptada para la integración e inclusión de esos alumnos.

\section{1 - Legislación Orientada A La Educación Especial}

Para dar inicio es fundamental señalar lo que dice el artículo tercero de la Constitución Política de los Estados Unidos Mexicanos (2008, pp. 6-7):

\footnotetext{
"Todo individuo tiene derecho a recibir educación. El Estado - Federación, estados, Distrito Federal y municipios- impartirá
} 
educación Preescolar, primaria y secundaria. La educación preescolar, primaria y secundaria conforma la educación básica obligatoria. La educación que imparta el estado tendrá a desarrollar armónicamente todas las facultades del ser bumano".

Con base a lo anterior, se define a la educación como la encargada de desarrollar las capacidades humanas de las personas, siendo estrictamente obligatoria para el al nivel básico, donde acceder y permanecer en la escuela se vuelve un proceso democrático, y a la vez un derecho. Por otra parte, dará respuesta a las necesidades de cada uno de sus educandos. Es decir, se tendrá presente, el proceso de equidad en el ingreso a la educación; haciendo énfasis en las acciones del sistema educativo para brindar las mismas oportunidades de aprendizaje y experiencias necesarias en el desarrollo fisico, intelectual y emocional pleno de cualquier presona.

Se considera necesario, dar a conocer parte de lo que se ha establecido en diferentes leyes puestas en marcha a partir de los cambios que se han dado a través de las demandas de una sociedad que está en constante cambio. Por tal motivo, se pretende dejar claro lo más relevantes en cuanto a integración e inclusión educativa se refiere.

Como parte los antecedentes políticos, es preciso señalar una de las leyes que han influido de manera fundamental en los procesos educativos de nuestro país. Nos referimos entonces a la Ley Federal de Educación de 1973. Por tanto, es necesario señalar como considera a la educación especial.

CAPITULO II Sistema Educativo Nacional.
ARTICULO 15. El sistema educativo
nacional comprende los tipos elemental, medio y
superior, en sus modalidades escolar y
extraescolar. En estos tipos y modalidades
podrán impartirse cursos de actualización y
especialización. El sistema educativo nacional
comprende, además, la educación especial o la de
cualquier otro tipo y modalidad que se imparta
de acuerdo con las necesidades educativas de la
población y las características particulares de los
grupos que la integran. (Secretaría de educación
Pública, 1973, p.3).

En esta ley se reconoce a la educación especial como una modalidad. Sin embargo, aunque ya hacía referencia a la atención de las NEE. No tenía bien definidos objetivos en cuanto integración educativa, Sin embargo, los objetivos del Estado se han enfocado en ir estableciendo más y mejores leyes surgidas de nuevas reformas, que son entendidas como cambios en nuestraa politica educativa. De tal manera, señalar el resultado de los propósitos que buscan el desarrollo educativo de la nación nos obliga a revisar lo que precede. Llegando así a lo que hoy se conoce como Ley General de Educación, Promulgada en Julio 1993. De tal 
manera, Cabe mencionar, que nos enfocamos en la parte que resalta el objetivo de la educación especial.

CAPITULO IV DEL PROCESO EDUCATIVO; SECCIÓN 1.- DE LOS TIPOS Y MODALIDADES DE EDUCACIÓN. ARTÍCULO 41.La ducación especial está destinada a personas con discapacidad, transitoria o definitiva, asi como a aquellas con aptitudes sobresalientes. Atenderá a los educandos de manera adecuada a sus propias condiciones, con equidad social incluyente y con perspectiva de género. Párrafo reformado DOF 12-06-2000, 17-04-2009, 28-01-2011. Tratándose de menores de edad con discapacidad, esta educación propiciará su integración a los planteles de educación básica regular, mediante la aplicación de métodos, técnicas y materiales específicos. Para quienes no logren esa integración, esta educación procurará la satisfacción de necesidades básicas de aprendizaje para la autónoma convivencia social y productiva, para lo cual se elaborarán programas y materiales de apoyo didácticos necesarios. Párrafo reformado DOF 12-062000, 28-01-2011.Para la identificación y atención educativa de los alumnos con capacidades y aptitudes sobresalientes, la autoridad educativa federal, con base en sus facultades y la disponibilidad presupuestal, establecerá los lineamientos para la evaluación diagnóstica, los modelos pedagógicos y los mecanismos de acreditación y certificación necesarios en los niveles de educación básica, educación normal, asi como la media superior y superior en el ámbito de su competencia. Las instituciones que integran el sistema educativo nacional se sujetarán a dichos lineamientos. (Secretaría de educación pública, 1993, p18)

Con base en lo anterior podemos darnos cuenta de la importncia que tiene este parte de la ley. Pues señala que la educación especial esta destinada para todos los alumnos que presenten Nee, con sisn discapacidad. Así mismo, por medio de esta educación se establce el objetivo que pretende atender las problematicas que presente estos alumnos, considerando el proceso necesario para que logren su integración y el máximo desempeño escolar, enfocandose en sus ritmos y estilos de aprendizaje y utilizando métos, técnicas y materiales especificos para su aprendizaje. Señalando tambien la responsabilidad de las autoridades educativas en establecer los lineamientos de la evaluación que se les aplicará a los alumnos en el diagnostico dentro de los niveles de educación básica, incluyendo tambien a la media superiror y superior.

Como se guimiento del proceso legislativo, podemos señalar que los cambios han progresado, estableciendose programas que han tomando en cuenta a la educación especial, haciendo énfasis en la discapacidad y sus repercuciones en nuestros alumnos, de tal manera, se han implementado y puesto en marcha acciones, las cuales se destacaremos en El Programa Nacional de Fortalecimiento de la Educación Especial y la Integración Educativa. 
En materia educativa El Programa Nacional de Fortalecimiento de la Educación Especial y de la Integración Educativa (2001-2006) es garantizar la equidad y mejorar la calidad del proceso y los resultados de las evaluaciones. Alcanzar la justicia educativa y la equidad es el primer objetivo estratégico establecido en el Programa Nacional del Sector; implicando un conjunto de acciones para revertir la desigualdad educativa y favorecer mediante mayores y mejores recursos a la población vulnerable de nuestro pais, de la que forma parte la población con discapacidad (Secretaría de Educación Pública, 2001-2006, p. 7).

Con lo aquí dispuesto nos es posible entender, que en nuestro país se han puesto en marcha alternativas en el ámbito de la Educación Especial, desarrollando prácticas educativas donde se implementen métodos, estrategias con el fin de brindar el acceso y asegurar la permanencia en las escuelas de los alumnos que presenten alguna discapacidad. Por tanto, la siguiente información expresa el objetivo del mismo.

El Programa Nacional de Fortalecimiento de la Educación Especial y de la Integración Educativa (2001-2006) constituye una respuesta del gobierno federal a las demandas y propuestas ciudadanas en materia educativa; establece la ruta que habremos de seguir para consolidar una cultura de integración que contribuya a la constitución de una sociedad incluyente donde todos los hombres y mujeres tengan las mismas oportunidades de acceder a una vida digna (Secretaría de Educación Pública, 2001-2006, p. 7).

La integración se toma en cuenta en diferentes ámbitos de índole, educativos, sociales y culturales, misma que tiene como meta favorecer el desarrollo emocional, intelectual y físico de las personas. Dependiendo del contexto en el que se sitúen. Por ende, la integración pasa a ser una ideología que busca crear un ambiente incluyente en donde se respete la diversidad, no solo de las personas con discapacidad, sino también considerando otras características como la raza, sexo y religión.

De acuerdo con lo que establece el PROGNAL, la integración surge como parte de la necesidad de dar respuesta a las demandas de la sociedad, que exige y necesita ser comprendida en cuanto a su participación dentro de un determinado grupo social, situándose en diferentes contextos. Donde se tengan las mismas oportunidades y la sana convivencia sea parte del proceso. Entendiendo que los actos discriminadores y racistas son lo contrario a lo que se denomina como sociedad incluyente. Reforzando esta idea de sociedad incluyente, nos enfocamos entonces a señalar lo que dice el Plan Nacional de Desarrollo, específicamente en la meta denominada México Incluyente. Enfocándonos en el plan de acción que dice: 
II.2. Plan de accion: integrar una sociedad con equidad, cohesión social e igualdad de oportunidades. Un México Incluyente plantea una politica social de nueva generación. Es decir, una política enfocada en alcanzar una sociedad de derechos ciudadanos y humanos plenos. En este sentido, se proponen políticas sociales que giran en torno al ciudadano, ubicándolo como un agente de cambio, protagonista de su propia superación a través de su organización y participación activa. La prioridad será integrar una sociedad con equidad, cohesión social e igualdad de opor- tunidades. Un México Incluyente busca consolidar plataformas de movilidad social que contribuyan a cerrar las brechas existentes entre diferentes grupos sociales y regiones del país. (Gobierno de la República, 2013-2018,p 53)

Estas acciones de tomar en cuenta a la sociedad y al ser humano como parte de ellal, así como señalar los fenómenos más presentes actualmente en cuanto a falta de equidad, obliga a las políticas a orientar disposiciones en donde el realce de los derchos humanos se haga parte fundamental para procurar erradicar las problematicas que impiden la igualdad de oportunidades en diversas situaciones. Por tanto, los grupos constituidos por los grupos de minorias que incluyen a grupos étnicos, personas con discapacidad, padecientes de pobreza extrema que no tienen oportunidades de acceder a diversos servicios de carácter público; como lo son la educación y la salud. se vuelven el objetivo principal de lo que el Plan Nacional de
Desarrollo. Sin embargo, reforzando el valor de los derechos humanos en la sociedad, la parte que consideramos importante señalar es la siguiente:

Se buscará asegurar la vigencia efectiva de los
derechos de las personas con discapacidad y
contribuir a su desarrollo integral, lo que
transita por su inclusión al mercado de trabajo y
la dinámica social, además de impulsar, con el
apoyo de los medios de comunicación y la
sociedad civil, estrategias que coadyuven a
transformar la actual cultura excluyente y
discriminatoria en una abierta a la tolerancia y
la diversidad. (Gobierno de la República, 2013-
2018, p. 53)

Claramente, el objetivo primordial es favorecer la integración de las personas con discapacidad en diferentes ámbitos, en este caso haciendo énfasis en el laboral y la participación social; ámbitos que se considera importante para el desenvolvimiento e independencia de cualquier individuo; está parte señala también la importancia del apoyo de diversos medios de comunicación para la difusión de información que tengan como meta; dar información acerca de la discapacidad y de esta forma impactar de manera significativa en las personas que la vean o escuchen, generando así, un proceso de sensibilización y un cambio de actitudes en nuestra sociedad. De esta manera, se propiciará una mejor calidad de vida para aquellos que presenten alguna condición física o psicológica que los limite hacer ciertas actividades. 
Por otra parte, específicamente en el ámbito de educación, nos enfocamos en como se ha favorecido el proceso escolar de los alumnos con algún déficit asociados o no a una discapacidad. Siendo preciso señalar el objetivo general que establecen las Normas Específicas de Control Escolar Relativas a la Inscripción, Reinscripción, Acreditación, Promoción, Regularización y Certificación en la Educación Básica.

Titulo 1: disposiciones generales. Capitulo 1 objeto, ámbito de aplicación, difusión e interpretación. 1,-Favorecer que las personas ejerzan su derecho constitucional a recibir educación básica (preescolar, primaria y secundaria); por lo que, queda probibida toda discriminación motivada por origen étnico o nacional, situación migratoria, sexo, edad, discapacidades, condición social, condiciones de salud, religión, opiniones, preferencias sexuales, estado civil o cualquier otra característica propia de la condición bumana que tenga por objeto anular o menoscabar los derechos y libertades de las personas en un marco de respeto a los derechos humanos. 2.- Servicios educativos en que aplican las normas de control escolar: Las presentes normas son aplicables a los servicios educativos del tipo básico, a las instituciones educativas públicas y particulares con autorización, incluyendo a la educación indígena, a la educación especial, así como a la atención de la población migrante y la que es impartida en albergues, centros de alto rendimiento, aulas multigrado, centros de atención múltiple, servicios educativos comunitarios que opera el Consejo Nacional de Fomento Educativo (CONAFE), y demás centros educativos. (Secretaría de educación pública, S.f, p 6)

Con base a lo que se establece, consideramos que el acceso y la permancia de los alumnos en la educación debe ser apegada a derecho, y con esto nos referimos a que debe ser justa, con iguales oportunidades para todos sin importar las condiciones de salud, religión, preferencia sexual, nacionalidad, etnia, sexo, edad o discapacidad. Entendiendo también que esta ley se aplicará en los niveles de educación básica, tomando en cuenta a la educación especial como parte fundamental del proceso.

De esta manera, se construye a la educación como una oportunidad para quienes ingresen y se ven inmersa en ella, ya que se pone de manifiesto el respeto a los derechos humanos. por otra parte, es interesante observar que resalta a la educación como un derecho, reforzando lo que dice el articulo tercero constitucional.

Además de los elementos ya mencionados, podemos tener en cuenta que nuestra sociedad es diversa, y que cada persona tiene caracteristicas diferentes, por tal motivo, esto permitirá dar paso a una ideología diferente entre quienes convivimos dentro de un determinado contexto. Consideramos que estos mandatos de carácter nacional, son y deveran ser los que den paso a una ideologia incluyente. En un proceso que quizá sea dificil, pero que poco a 
poco se capaz de eliminar la segregación y discriminación a cualquier grupo de personas. Facilitando el ingreso a la escuela y la permanencia en ella.

Por otra parte, Como prueba de los avances que se han tenido dentro de la educación especial, es preciso señalar lo que estable la Ley General Para la Atención y Protección a Personas Con la Condición del Espectro Autista.

CAPÍTULO I Disposiciones Generales. Articulo 2. La presente Ley tiene por objeto impulsar la plena integración e inclusión a la sociedad de las personas con la condición del espectro autista, mediante la protección de sus derechos y necesidades fundamentales que les son reconocidos en la Constitución Política de los Estados Unidos Mexicanos y en los tratados internacionales, sin perjuicio de los derechos tutelados por otras leyes $u$ ordenamientos.

CAPÍTULO II De los Derechos y de las Obligaciones. Sección Primera De los Derechos Artículo 10. Se reconocen como derechos fundamentales de las personas con la condición del espectro autista $y / 0$ de sus familias, en los términos de las disposiciones aplicables, los siguientes:

I. Gozar plenamente de los derechos bumanos que garantiza la Constitución Política de los Estados Unidos Mexicanos y las leyes aplicables;

II. Recibir el apoyo y la protección de sus derechos constitucionales y legales por parte del Estado Mexicano-federación, entidades federativas y municipios-;

III. Tener un diagnóstico y una evaluación clínica temprana, precisa, accesible y sin prejuicios de acuerdo con los objetivos del Sistema

Nacional de Salud;

IV. Solicitar y recibir los certificados de evaluación y diagnóstico indicativos del estado en que se encuentren las personas con la condición del espectro autista;

$V$. Recibir consultas clínicas y terapias de habilitación especializadas en la red hospitalaria del sector público federal, de las entidades federativas y municipios, así como contar con terapias de habilitacion;

VI. Disponer de su ficha personal en lo que concierne al área médica, psicológica, psiquiátrica y educativa, al igual que de los certificados de babilitación de su condición, al momento en que les sean requeridos por autoridad competente;

VII. Contar con los cuidados apropiados para su salud mental y física, con acceso a tratamientos y medicamentos de calidad, que les sean administrados oportunamente, tomando todas las medidas y precauciones necesarias;

Ser inscritos en el Sistema de Protección Social en Salud, conforme a lo establecido en la Ley General de Salud;

IX. Recibir una educación o capacitación basada en criterios de integración e inclusión, tomando en cuenta sus capacidades $y$ potencialidades, mediante evaluaciones pedagógicas, a fin de fortalecer la posibilidad de una vida independiente;

$X$. Contar, en el marco de la educación especial a que se refiere la Ley General de Educación, con elementos que faciliten su proceso de integración a escuelas de educación regular.

Esta ley pone en de manifiesto la atención de las personas que padecen autismo. Es un ejemplo claro de los avances que se han tenido en 
educación especial, ya que establece sus derechos en su atención clínica, psicológica y educativa que merecen y ameritan debido a su condición. Es preciso también hacer énfasis en la parte que hace mención al proceso de integración en las escuelas regulares, donde según la ley deben contar con los elementos necesarios para que esto suceda. Consideramos que esta ley es de vital importancia, pues pone de manifiesto los derechos de las personas con espectro autista y se enfoca en lograr la superación de actos discriminadores hacia estas personas.

\section{2 - Discapacidad Y Nesecidades Educativas Especiales (Nee)}

En este epígrafe dejaremos claros algunos conceptos que se relacionan con la integración y la inclusión educativa. Entender que origina las necesidades educativas especiales y cómo se clasifican, se vuelve fundamental. Por otra parte, dar respuesta algunas interrogantes dará un complemento a la información. Definiremos entonces: ¿Qué es la discapacidad?, ¿Cuándo se considera que un niño tiene discapacidad?, ¿Por qué se encuentra en desventaja ante sus demás compañeros?, ¿Qué son las barreras para el aprendizaje y la participación?

\section{La ley para protección de los derechos de las} niñas, niños y adolescentes (2008), en el capitulo IX, articulo 29.- para efectos de esta ley, se considera persona con discapacidad a quien padezca una alteración funcional física, intelectual o sensorial, que le impida realizar propia de su edad y medio social, y que implique desventaja para su integración familiar, social, educacional o laboral (El Congreso de los Estados Unidos Mexicanos, 2000 p.20)

Si bien lo anterior da hincapié a definir la discapacidad como una condición que afecta a los niños(as) y jóvenes en su interacción, relación y convivencia en los contextos más importantes: familiar, social y escolar; teniendo presente que tendrán limitantes para realizar actividades que otros puedan efectuar con facilidad. Por tanto, habrá que considerar a la escuela como la vía principal que facilite su educación, adecue la infraestructura promueva espacios, materiales educativos y pedagógicos que contribuyan a su proceso de integración e inclusión educativa. Siendo importante resaltar que las desventajas que presenta cualquier individuo en el entorno que se desenvuelve, se denomina minusvalía. Sin embargo, para entender mejor los conceptos de discapacidad y minusvalía recurrimos a lo que se establece a continuación.

- Deficiencia: "Anomalía de la estructura corporal y de la apariencia. Con pérdida o anormalidad de la función de algún órgano o sistema. Cualquiera que sea su causa". Discapacidad: "Refleja la consecuencia de la deficiencia desde el punto de vista del rendimiento funcional y de la actividad del individuo. Las discapacidades representan los trastornos a nivel de la persona". - Minusvalía: "Hace referencia a las desventajas que experimenta el individuo como consecuencia de las discapacidades. Las minusvalias 
reflejan la interacción y adaptación del individuo al entorno Y viceversa". (Cardona Martfn, 2,001, pp.15-18).

Considerando estos términos hacemos énfasis en lo que mencionan Michael W \& James A (2000). Las escuelas democráticas, como la democracia misma, no se producen por causalidad. Se derivan de intentos explícitos de los educadores de poner en vigor las disposiciones y oportunidades que darán vida a la democracia. De acuerdo con este planteamiento, consideramos que, los maestros crearán estructuras y procesos democraticos mediante los cuales se transforme la vida en la escuela, y por otra parte, diseñaran un curriculum que aporte experiencias a los jóvenes.

De igual manera, si tomamos en cuenta la descripción de las limitantes que pudiera originar la discpacidad. Cuando la escuela no propicia espacios adecuados, no cuenta con personal capacitado, o simplemente impide el acceso de los alumnos con discapacidad. Nos enfrentamos a un fenómeno que no permite un proceso de integración e inclusión necesario, para que los alumnos se desenvuelvan de manera plena y adquieran un desarrollo de sus capacidades, tomando en cuenta sus individualidades. En ocaciones esto trasciende a diversos contextos, creando un ambiente de discriminación y segregación.

Las barreras para el aprendizaje y la participación son todos aquellos factores del contexto que dificultenn o limiten el pleno acceso a la educación y a las oportunidaes de aprendizaje de niñas, niños y jóvenes. Aparecen en relación con su interacción en los diferentes contextos: social, político, institucional, cultural y en las circunstancias sociales y económicas. Desde el enfoque de la educaion inclusiva, este concepto rebasa al de nesecidades educativas especiales ya que se centra en la interacción con el conexto y no como un problema inherente al alumno. (Comisión de Política Gubernamental en México de Derechos Humanos, s.f, p. 5)

Aunque el término anterior es parte de la actualización de la educación especial, en los procesoso de integración e inclusión se habla de las nesecidades educativas especiales (NEE). Revisando diversas fuentes nos encontramos que coinciden, al decir que estas se dan cuando un niño, a comparación de sus compañeros, tiene mayores dificultades para acceder a los aprendizajes. Sin embargo, para ser más precisos consideramos importante señalar la siguiente información.

Alumno con nesecidades educativas especiales: Aquel o aquella que presentan un desempeño escolar significativamente distinto en relación con sus compañeros de grupo. Por lo que requiere que se incorporen a su proceso educativo mayores $y / 0$ distintos con el fin de lograr su participación y aprendizaje, y alcanzar asi los propósitos educativos.

Estos recursos pueden ser profesionales (personal de educacón especial, de otras instancias gubernamentales o particulares), materiales. (mobiliario especifico, protesis, material didáctico), y curriculares (adecuasiones en la metodología, contenidos, propósitos y evaluación). Las nesecidades educativas especiales que presente el alumno no 
pueden ser temporales o permanentes y estar asociadas o no a una discapacidad o aptitudes sobresalientes. (Comisión de Politica Gubernamental en México de Derechos Humanos, s.f, p. 10)

Hablando de necesidades educativas especiales, algunos autores le han dado una definición con base a sus investigaciones. Por tanto, se necesita entender cómo es que se clasifican y como repercuten en los alumnos que las presentan. Por tal motivo, revisando antecedentes de España se encontró lo siguiente:

El grupo de sujetos considerados de necesidades educativas especiales "incluye a quienes tienen impedimentos físicos o sensoriales, a aquellos que comparados con los pares de su edad tienen dificultades en su aprendizaje o al comunicarse, como también a aquellos cuya conducta no puede ser aceptada sin problemas en las aulas o escuelas regulares.
Lo que estos niños tienen en común es una mayor o menor necesidad de que se les brinde una oferta educativa especial que esté por encima de la que la generalidad de las escuelas ofrecen a la mayoría de los estudiantes. (Hegarty, 1994, $p$. 138)

En la siguiente tabla quedó expresada la clasificación que hiso Hegarty sobre necesidades educativas especiales, mismas aún se siguen utilizando dentro de la educación especial, para entender y explicar por qué los alumnos tienen problemas en su aprendizaje, ya que a partir de esto es posible darles una respuesta pedagógica más adecuada. Por tanto, esta información es fundamental para quienes se ven involucrados en el trabajo de integrar e incluir a los niños y jóvenes que requieren de mayores esfuerzos para acceder a los aprendizajes.

\begin{tabular}{|c|c|c|}
\hline Personales & Escolares & Sociales \\
\hline $\begin{array}{l}\text { Deficiencia mental } \\
\text { Deficiencia física } \\
\text { Deficiencia sensorial }\end{array}$ & $\begin{array}{l}\text { Ignorar el ritmo de aprendizaje del } \\
\text { alumno. } \\
\text { Objetivos inadecuados } \\
\text { Contenidos inadecuados }\end{array}$ & Deficiente aprendizaje \\
\hline $\begin{array}{l}\text { Audición y sordera } \\
\text { Hipoacusia } \\
\text { Visión } \\
\text { Ambliopía } \\
\text { Alteraciones } \\
\text { Neuralgias } \\
\text { Trastornos del desarrollo }\end{array}$ & $\begin{array}{l}\text { Metodología } \\
\text { Actividades inadecuadas } \\
\text { Pasividad del alumno } \\
\text { Deficiente- } \\
\text {-Intercomunicación entre profesor y } \\
\text { alumno } \\
\text { Historial de aprendizaje } \\
\text { Desajustado (temporeros, deportistas, } \\
\text { artistas...) }\end{array}$ & $\begin{array}{l}\text { Desequilibrados afectivos } \\
\text { Y emocionales. } \\
\text { Nivel sociocultural } \\
\text { Familiar }\end{array}$ \\
\hline $\begin{array}{l}\text { Ontológicas } \\
\text { Dificultades internas al alumno }\end{array}$ & $\begin{array}{l}\text { Didácticas } \\
\begin{array}{l}\text { Dificultades en el proceso de } \\
\text { enseñanza-aprendizaje }\end{array}\end{array}$ & $\begin{array}{l}\text { Ecológicas } \\
\text { Dificultades del contexto }\end{array}$ \\
\hline
\end{tabular}

52 MUJICA, D.Y.R.; FELICIANO, T.M.; GUTIÉRREZ, M.Z.: Discusión Teórica Respecto A La Atención De Las Nee (Necesidades Educativas Especiales) En La Legislación Educativa Mexicana. 
Con base a la anterior clasificación de Hegarty. Es posible observar y analiza $\mathrm{r}$ Las causas de las NEE, las cuales son diversas, así como sus repercusiones en el aprendizaje. Cada uno de estos factores implicados en el desarrollo físico, emocional, e intelectual del niño, deben ser atendidos por los padres de familia como primera agencia de socialización y en segunda instancia por los maestros en el trabajo pedagógico dentro de la escuela. Los procesos de integración, inclusión y participación en el aula, cambiaran de manera significativa la vida de los alumnos que en algún momento sufrieron situaciones de discriminación y segregación.

\section{3 - Política Educativa Orientada En El Margen De La Educación Especial}

Antes de definir que son las políticas educativas, tendremos que entender el significado de política, es decir, relacionaremos los términos para hacer más fácil la comparación y la relación. Por tanto, consideramos importante recurrir a la siguiente cita:

Son muchas y distintas las repuestas que, desde distintas perspectivas, se han dado sobre el concepto de la politica: para unos, la política consiste esencialmente en la lucha por el poder dentro de una determinada politica, mientras que para otros en la acción colectiva dirigida a la consecución del bien común. Por ejemplo. En el mundo académico, en concreto, se suelen entender por politica tres cosas: a) el marco institucional y las condiciones generales en el que se desenvuelve la acción política: b) los contenidos concretos de la acción política, es decir, las acentuaciones en los ámbitos determinados en los que se realizan los programas politicos (educación, sanidad, comunicaciones, etc.), y c) el proceso global en el que se conforman la lucha por el poder y la influencia. A estas tres divisiones académicas del estudio de la politica se puede añadir que la política consiste también en un tipo de saber, que pretende contribuir a las formulaciones de máxima de actuación para los gobernantes y a los ciudadanos en general. (Franzé, 2004, p. 13-14)

Al hablar de política, entendemos que hay varias definiciones. Sin embargo, la que nos interesa es aquella que hace referencia a las acciones del Estado, para contribuir en la mejora de la sociedad. Entendiendo así, que la educación se ve involucrada como una de las prioridades del sistema educativo, comprendida como un derecho destinado a los alumnos y alumnas del país. De tal manera, definimos a política educativa en la siguiente cita:

Las politicas educativas son todas aquellas leyes impuestas por el estado para garantizar que la educación se aplique de manera óptima a la sociedad. Estas leyes se forjan de acuerdo a las necesidades históricas del país en el que se promueven, es decir, según sus ideales y principios. Las políticas educativas son creadas con el fin de administrar y aplicar con beneficio para la sociedad, todos los recursos que el Estado destine para este fin. Las políticas educativas tienen el objetivo de tratar y resolver todos los problemas a los que la educación se enfrenta, por ejemplo, la economía, la cultura o la burocracia y transformarlos en la maximación de los logros de los objetivos como la calidad, la eficacia y la eficiencia. (Zamara, s.f, p. 3) 
Con base a esto, entendemos que la política se relaciona directamente con las leyes. Estas serán las que determinen las acciones para favorecer cambios en el sistema educativo. Claro, tomando en cuenta distintos factores de carácter social y economico.

Si bien menciona Neville \& Tim (1995), a la política se llega mediante unos procesos racionales y mediante la persecución de unos objetivos que, cuando se logran, formarán parte de la actitud vital de la escuela. Por tanto, se entiende este planteamiento como la aplicación de política educativa a las escuelas de educación básica en nuestro país. De acuerdo con Zamara (s.f), las políticas educativas en México están orientadas a mejorar la calidad de la educación, se enfatiza el cambio curricular, organizacional, constitucional, así como mejorar el nivel de los maestros.

Los aspectos relacionados con política educativa son importantes para determinar cuál es el impacto que tienen en nuestras escuelas, y de qué manera influye en las prácticas de nuestros docentes que se dedican a la educación especial en las escuelas regulares. Por tal motivo, es factible considerar los siguientes planteamientos, ¿cómo la política educativa orienta y beneficia a las escuelas que consideran a la integración y a la inclusión como uno de sus principales objetivos?, ¿Cómo asumen los profesores su labor en una escuela integral?

Para darle una respuesta a dichos planteamientos se considera relevante decir que este trabajo arduo llamado escuela integral, se realiza de manera colectiva, observando las necesidades de la institución y de todos los alumnos que se encuentran en ella, se trata de reconocer y actuar ante la problemática de manera gradual, abatirla mediante una orientación que implique y logre beneficiar a los alumnos, en especial con aquellos que tienen un déficit en su proceso de aprendizaje y así logren sentirse emocional, física y psicológicamente bien en el entorno donde se desenvuelven. Según Neville \& Tim (1995), se define una política de escuela integral, mediante la creación de una plataforma para reiterar los principios, anima a los profesores a pensar más profundamente acerca de la naturaleza de la empresa educativa en la que estan implicados.

Esta idea de política asume que los maestros reconocen la integración como un principio, para brindar la atención de los alumnos con nesecidades educativas especiales, tomando en cuenta que no sólo los maestros son responsables de esto, también los demás actores de la escuela que participan para hacer una escuela integral.

Al referirnos al proyecto de integración educativa nos enfrentamos a la existencia de conceptos y categorias, objetivos, metas, definición de roles y 
estrategias preestablecidas, definitorias desde lo oficial, de dicho proyecto. Sin embargo, como marco normativo de política educativa actual, sin duda, incide sobre los actores sociales que viven y desarrollan cotidianamente las acciones propias de su realización, asi como al sentido que le atribuyen a esta práctica; por lo que resulta importante dimensionar las experiencias de los docentes, los directivos, las mismas personas con NEE, sus familiares, $y$ demás personal que trabaja en esta tarea. Indagar sus perspectivas al respecto resulta indispensable para que la labor de investigación pueda reconstruir sus puntos de vista y contar con un punto de vista de la integración educativa desde quienes se encuentran directamente involucrados en ella. Miramontes, Andrés, y Emily, 2004, p.9).

Retomando información que se ha revisado. Podemos decir con seguridad que en nuestro país se han tomado acciones para favorecer el acceso de los alumnos con NEE, con o sin discapacidad a la educación básica, que comprende los niveles de preescolar, primaria y secundaria. Para ello, como ya se había mencionado, lo establecido en leyes puestas en marcha tienen la finalidad de evitar la desigualdad de oportunidades de nuestras poblaciones menos favorecidas y por el contrario busca garantizar el derecho a la educación expresado como la igualdad de oportunidades para el acceso, la permanencia y el logro educativo de todos los niños y jóvenes del país.
Capitulo X. Del derecho a la educación. Art 32.- Niñas, niños y adolescentes tienen derecho a una educación que respete su dignidad y les prepare para la vida en un espiritu de comprensión, paz, tolerancia en los términos del art. $3^{\circ}$ de la constitución. Las leyes promoverán las medidas necesarias para que: a) se les proporcione la atención educativa, que por su edad, madurez y circunstancias especiales requieren para su pleno desarrollo; b) se evite la discriminación de las niñas y las adolescentes en materia de oportunidades educativas. Se establecerán los mecanismos que se requieren para contrarrestar las razones culturales, económicas o de cualquier otra indole, que propicie dicha discriminación; c) las niñas, niños $y$ adolescentes que posean cualidades intelectuales por encima de la media, tengan derecho a una educación acorde a sus capacidades, asi como contar con las condiciones adecuadas que les permita integrarse a la sociedad; d) se impulse la enseñanza y el respeto de los derechos humanos. En especial la no discriminación y de la convivencia sin violencia; e) se prevén mecanismos de participación democrática en todas las actividades escolares, como medio de formación ciudadana. (Diario Oficial de la Federación, 2000 p.22).

A continuación se muestran otros aportes que han incidido en nuestra política educativa para favorecer las posibilidades de acceso a la escuela regular. Organizaciones que han realizado declaraciones, hoy son de gran impacto en los propósitos de nuestra educación que intenta ser integradora, respetando las individualidades de 
cada persona. Tomando en cuenta a la discapacidad y sus repercusiones en el aprendizajes de niños y adolescentes que presentan NEE.

La declaración mundial de Educación para todos (UNESCO, 1990), establece; que cada persona deberá contar con posibilidades educativas para satisfacer sus necesidades de aprendizaje básico; mismas que abarcan tanto las berramientas esenciales (lectura, escritura, expresión oral, cálculo, solución de problemas) como los contenidos básicos (conocimientos teórico-prácticos, valores y actitudes) y que, desde este orden institucional, se supone que son fundamentales para que los seres humanos puedan sobrevivir, desenvolver completamente sus capacidades, vivir y trabajar con dignidad, participar de forma plena en el desarrollo, mejorar la calidad de vida, tomar decisiones fundamentadas y continuar aprendiendo.

Como parte de esta masificación se despliega la propuesta que conocemos como Proyecto de Integración Educativa (PIE). Actualmente, en el ámbito mundial, la educación básica institucional está suscrita a una política de integración educativa que es resultado del compromiso de Educación para todos y del reconocimiento de impartir enseñanza a los niños con necesidad educativa especial (NEE), adquirida en la conferencia mundial sobre necesidades educativas especiales, celebrada, en 1994, en Salamanca, España. Mares Miramontes \& Ito Sugiyama, 2004.p, 904)

Lo anterior reafirma el derecho de toda persona a la educación, pues será la vía para que todas las personas desarrollen sus capacidades humanas. De tal manera, retomaremos entonces la política destinada a la integración educativa, enfocándonos en el PROGNAL; mismo que ha puesto en marcha acciones destinadas a este fin.

En el Programa Nacional de Educación 2001 2006 reconoce la necesidad de poner en marcha acciones decididas por parte de las autoridades educativas para atender a la población con discapacidad. Asimismo se establece como uno de los objetivos estratégicos de la política educativa alcanzar la justicia y equidad. Entre las líneas de acción destacan las siguientes: establecer el marco regulatorio - asi como los mecanismos de seguimiento y evaluación- que babrá de normar los procesos de integración educativa en todas las escuelas de educación básica del país; garantizar la disponibilidad, para los maestros de educación básica, de los recursos de actualización y apoyos necesarios para asegurar la mejor atención de los niños y jóvenes que requieren de educación especial, además de establecer lineamientos para la atención a niños y jóvenes con aptitudes sobresalientes. (Secretaría de Educación Pública, 2001-2006,P.10)

En resumidas palabras el objetivo principal del PROGNAL, es garantizar el derecho a la educación expresado como la igualdad de oportunidades para el acceso, la permanencia y el logro educativo de todos los niños y jóvenes del país en la educación básica que presentan NEE, con o sin discapacidad.

4 - La Integración E Inclusión De Alumnos Con Nee En La Escuelas Regulares 
Hasta el momento nos hemos enfocado en los niños que presentan Necesidades Educativas Especiales en las escuelas regulares. Sin embargo, ahora describiremos el trabajo de los maestros de educación especial en la atención de estos alumnos con NEE, aspecto que consideramos fundamental para analizar el proceso de integración e inclusión educativa. Por tanto, antes de empezar, consideramos pertinente plantear la siguiente interrogante; ¿Qué es un maestro de educación especial?

Los maestros de educación especial (también conocidos como educadores especiales) tienen entrenamiento especializado para trabajar con estudiantes que tienen discapacidades de aprendizaje, comportamiento, emocionales, y/o físicas. Un maestro de educación especial principalmente trabaja con estudiantes que califican (basado en una serie de evaluaciones) para asistencia de educación especial. Educadores especiales trabajan en diferentes ambientes dependiendo en la necesidad de sus estudiantes y preferencias de su distrito escolar. Algunos educadores especiales tienen su propia aula escolar (por ejemplo: cuarto de recursos). Ellos sacan a los estudiantes de su aula regular $y$ asisten a estos estudiantes con horas especificas durante el día escolar y con sus necesidades especializadas de aprendizaje. Otros trabajan en el aula de educación regular apoyando a los estudiantes con necesidades especiales. Algunos educadores especiales tienen un grupo de estudiantes con discapacidades más complejas de comportamiento, emocional, de aprendizaje y/o físicas en un aula escolar independiente. Las necesidades de estos estudiantes son más grandes y requieren la asistencia adicional de maestros calificados y sus asistentes. (Becky L. Spivey, 2009, p 1)

El arduo trabajo que implica ser maestro de educacion especial, se relaciona directamente con un proceso donde se pretende ayudar aquellos alumnos con problemas de aprendizaje y con más énfasis en los que presentan alguna discapacidad, por lo tanto, los maestros buscan que alcancen los objetivos curriculares; toman en cuenta las adecuaciones curriculares necesarias y se apoyan con la participación de personal capacitado para dar respuestas a las problematicas que se pudieran presentar en la esculela, en cuestiones pedagógicas principalmente, estando concientes de las caracteristicas de sus alumos y respetando sus estilos y ritmos de aprendizaje. Sin embargo, se nesecitan espacios adecuados en donde se lleve acabo esta enseñanza, que tiene como finalidad brindar el acesoramiento al aula regular y reforzar el aprendizaje del alumno que lo requiere.

De acuerdo con la Politica de integración (UNESCO, 1994), el objetivo es proporcionar las condiciones que permitan la inserción de los niños con NEE al espacio social en general. Esta situación los coloca en el centro de la práctica educativa en tanto que, siendo el currículo regular su contexto de enseñanza aprendizaje, el docente habrá de realizar las adecuaciones pedagógicas necesarias para que superen las dificultades de aprendizaje, enfatizando en las posibilidades de desarrollo y consiguiente integración social.(Mares Miramontes \& Ito Sugiyama, 2004. P, 905). 
Es por esto que la política de integración nos habla del acceso a los niños(as) a una educación de calidad, donde se practiquen los métodos, estrategias y técnicas que beneficien al desarrollo físico y emocional en los diferentes espacios de la escuela. Será el docente entonces, quién se encargue de realizar el trabajo pedagógico, con la finalidad de que los alumnos tengan la oportunidad de acceder a los aprendizajes.

Con base en lo anterior planteamos la siguiente interrogante: ¿Cuál es la labor de los maestros ante las NEE?

\section{Tomando en cuenta el término de necesidades educativas especiales. Es importante señalar que los maestros pueden ver, pues, que la manera en que responden a las necesidades y dificultades de los niños depende, no sólo de los conocimiento sobre los niños y los grupos en los que se mueven, sino también de nuestro grado de comprensión acerca de nosotros mismos y acerca de nuestras propias reacciones a toda la gama de necesidades educativas especiales de los niños, especialmente si se manifiestan en un comportamiento perturbador o anormal. (Gerda Hanko, 1993.p. 88)}

Enendiendo lo que expone el autor, podemos decir que el docente por medio de la experiencia que adquirirá en la atención de niños con Nee, desarrollara habilidades y tomará conciencia de lo que implica dar respuesta a las problematicas que pueda presentar un alumno. De tal forma, sus conocimientos se ampliaran y los métodos que emplen serán cada vez mejores. Quizás en este trabajo se presenten un sinfín de dificultades, pero ahí se pondra en práctica los conocimientos del maestro ante las situaciones inesperadas que algún momento se pudieran presentar.

La integración educativa ha cambiado las ideologías de la escuela, siendo una alternativa para los niños con NEE. El PROGNAL describe a la integración como un proceso.

La integración educativa es el proceso que implica que los niños, las niñas y los jóvenes con necesidades educativas especiales asociadas con alguna discapacidad, con aptitudes sobresalientes o con otros factores estudien en aulas $y$ escuelas regulares, recibiendo los apoyos necesarios para que tengan acceso a los propósitos generales de la educación. Para fortalecer el proceso de integración educativa es necesario contar con la participación decidida de todas las autoridades educativas, especialmente de los responsables de la educación inicial, preescolar, primaria, secundaria y especial, asi como de los supervisores y directores de las escuelas, los maestros de grupo, el personal de apoyo de las escuelas, los padres y las madres de familia y el personal de educación especial. (Secretaría de Educación Pública , 2001-2006. p 23)

La integración pone en marcha distintos aspectos, que se deben tomar en cuenta para que se lleve a cabo con éxito. Por tanto, al ser uno de los principios de la educación especial, describe los siguientes pasos:

La integración educativa considera principalmente cuatro aspectos:

a) La posibilidad de que los niños y las niñas con necesidades educativas 
especiales aprendan en la misma escuela y en la misma aula que los demás niños.

b) Ofrecer a los niños y a las niñas con necesidades educativas especiales todo el apoyo que requieran, lo cual implica realizar adecuaciones curriculares para que puedan ser satisfechas las necesidades específicas de cada niño.

c) La importancia de que el niño, los padres y las madres y/o el maestro de grupo reciban el apoyo y la orientación necesaria del personal de educación especial.

d) Que la escuela regular en su conjunto asuma el compromiso de ofrecer una respuesta adecuada a las necesidades educativas especiales de los niños y las niñas. (Secretaría de Educación Pública , 2001- 2006, p. 23)

Por otra parte, en la actualización de conceptos se habla de inclusión educativa, algunos investigadores la definen la de la siguiente manera.

El concepto de inclusión hace referencia a que "Todos los niños/as y jóvenes del mundo, con sus fortalezas $y$ debilidades individuales, con sus esperanzas y expectativas, tienen el derecho a la educación. No son los sistemas educativos los que tienen derecho a cierto tipo de niños/as. Es por ello, que es el sistema educativo de un país el que debe ajustarse para satisfacer las necesidades de todos los niños/as y jóvenes" (B. Lindqvist, UN-Rapporteur, 1994). De manera que es necesario eliminar las clasificaciones, la etiquetación, la discriminación y considerar a la diferencia como una característica común a todas las personas; es decir lo que nos hace iguales es ser diferentes. (Barraza Macias \& Cárdenas Aguila, 2014, p. 38).
Con lo anteriror podemos entender que todos los niños y niñas de nuestro pais, considerando sus dificultades y aptitudes, tendrán derecho a la educación. Donde el sistema educativo se adaptará a sus necesidades y tendrá como objetivo satisfacer la igualdad de oportunidades sin distinción o segregación por raza, color, sexo, religión o presentar alguna discapacidad.

Por otra parte, tambien es un proceso que considera varios pasos a seguir y se describen a continuación (Barraza Macías \& Cárdenas Aguila, 2014, p. 38).

1. Iniciación del proceso del índice: se trata de constituir un grupo coordinador, sensibilización de la escuela respecto al Índice, explorar el conocimiento del grupo, prepararse para usar los indicadores y las preguntas y prepararse para trabajar con otros grupos; todo esto con una duración de medio trimestre.

2. Exploración y análisis de la escuela: consiste en la exploración del conocimiento del personal y de los miembros del Consejo Escolar, exploración del conocimiento del alumnado, exploración del conocimiento de las familias y de los miembros de la localidad y decisión de las prioridades a desarrollar. Se desarrolla durante un trimestre.

3. Elaboración de un plan de desarrollo de la escuela con una orientación inclusiva: se refiere a la introducción del Índice en el proceso de planificación de la escuela y la introducción de las prioridades en el plan de desarrollo de la escuela. Se realiza durante medio trimestre.

4. Implementación de los aspectos susceptibles de desarrollo: consiste en 
poner en práctica las prioridades, lograr el desarrollo sostenido y el registro del progreso. Se trabaja en forma continua.

5. Revisión del proceso seguido en el índice: esta etapa se refiere a la evaluación de los cambios, a la revisión del trabajo realizado con el Índice y a la continuación del proceso del Índice. También se trabaja en forma continua. Para lograr que una escuela se inicie en la inclusión es necesario atender a 3 dimensiones: crear culturas inclusivas, elaborar políticas inclusivas y desarrollar prácticas inclusivas. En lo que se refiere a las características de una escuela inclusiva, Climent Giné propone una serie de condiciones para una escuela inclusiva: $\square$ Trabajo colaborativo entre el profesorado, consiste en: - Desarrollar la intervención conjunta de dos profesores en el aula. - Planificación conjunta de las unidades de programación. - Incremento de la ayuda mutua. - Mejora de la conciencia de equipo docente. Promoción de la autoestima a partir de la colaboración.

Cabe mencionar que existe también un dilema entre los conceptos de integración e inclusión educativa. Lo que pretendemos es analizar su relación que tienen dentro de los cambios más recientes en educación. Por tanto, dejar en claro que es inclusión educativa y como es que ha sido una ideología que forma parte de la educación especial, se vuelve fundamental.

El debate sobre la educación inclusiva y la integración no se refiere a una dicotomía entre políticas y modelos de integración e inclusión - como si se pudiera integrar sin incluir o incluir sin integrar -, sino más bien a determinar en qué medida se está avanzando en el entendimiento de que toda escuela tiene la responsabilidad moral de incluir a todos y cada uno. (Unesco, 2008,p. 10)

Este nuevo concepto implica más que sólo actualizacion, se convierte en una aspiración que pretende mejorar la calidad educativa de los niños y jóvenes con NEE, con o sin discapacidad en las escuelas regulares. Teniendo como idelogía la atención en y para la diversidad, formulando tecnicas, estrategias y metodologías que sean capaces de propiciar un ambiente apropiado, según la condición del o de los alumnos que lo requieran.

la problemática de la diversidad implica un cambio radical de la escuela y a travez de ellas se busca democratizar las relaciones sociales en un contexto sumamente complejo y con altos coeficientes de marginalidad. Sin embargo, debemos relacionar la diversidad con lo que la escuela debe hacer para lograr la inclusión de sus alumnos. (Boggino \& de la Vega, 2006 p. 24)

Con esto se reconoce que la escuela se enfrenta a diferentes situaciaciones, donde se pretende realizar un proceso educativo democratico, combatiendo los fenomenos de marginalidad. De esta forma, se favorecerá la inclusión de los alumnos, en donde tambien se favorecerá la socialización.

La escuela, asu vez será concebida como una institución abieta, democrática y flexible, capaz de promover estrategias institucionales que permitan el aprendizaje de acuerdo a las caracteristicas, ritmos, y nesecidades de los dstintos grupos sociales, así como tambien aseguren su plena inclusion social. (Boggino \&o de la V ega, 2006, p.25) 
Con base a lo anteriror podemos decir que es la escuela busca adapatarse a los alumnos, entendiendo que esta será flexible, comprensiva con aquellos que presentan nesecidades educativas especiales. Considerando las caracteristicas de cada alumno, estilo y ritmos de aprendizaje.

Por consigiente la educación que se define como inclusiva, según la unesco se define de la siguiente manera:

La educación inclusiva puede entenderse como un principio rector destinado a alcanzar niveles razonables de integración escolar de todos los estudiantes. En el contexto de una visión más amplia de la integración, la educación inclusiva supone la formulación y aplicación de una vasta gama de estrategias de aprendizaje que respondan precisamente a la diversidad de los educandos. En este sentido, los sistemas educativos deben responder a las expectativas y necesidades de los niños y jóvenes teniendo en cuenta que la capacidad de ofrecer oportunidades reales de aprendizaje sobre la base de un esquema "rígido" de integración es muy limitada. (Unesco, 2008, p. 10)

Este concepto se convierte en un paradigma que pretende mejorar la educación especial y las prácticas de los docentes, que tienen la responsabilidad de dar respuesta a las individualidades, rescatando las capacidades de los alumnos en lugar de acentuar sus discapacidades. La siguiente información describe a la inclusión y lo que pretende hacer con la escuela, tranformandola en un lugar donde la discriminación y la segreagación dejen de ser parte de las prácrticas educativas. Lo siguiente, refuerza esta postura:

En los últimos quince años aproximadamente, el concepto de educación inclusiva ba evolucionado hacia la idea de que todos los niños y jóvenes, no obstante las diversidades culturales, sociales $y$ de aprendizaje, deberían tener oportunidades de aprendizaje equivalentes en todos los tipos de escuelas. El centro de atención se sitúa en la creación de entornos inclusivos, lo cual implica: a) el respeto, la comprensión y la atención a la diversidad cultural, social $e$ individual (respuesta de los sistemas educativos, escuelas $y$ docentes a las expectativas $y$ necesidades de los alumnos); b) el acceso en condiciones de igualdad a una educación de calidad; y c) la estrecha coordinación con otras políticas sociales. En todos estos puntos se deben tomar en cuenta las expectativas y las demandas de las partes interesadas y los actores sociales (Unesco, 2008, p10).

El término de educación inclusiva ha tenido auge los últimos, la Unesco ha propuesto la definición del concepto, mismo que ha tenido como finalidad cambiar las prácticas educativas de los docentes. Se relaciona con los intereses de todos aquellos que se vean involucrados en el proceso; maestros, padres de familia y alumnos. Todos estos considerados como actores sociales en la escuela. 
5 - La Evaluación Psicopedagógica En Educación Especial (Trabajo En El Aula)

Refiriendonos especificamente a educación especial, damos hincapie a lo que se refiere al trabajo dentro del aula con los alumnos que presentan Nesecidades Educativas especiales, con o sin discapacidad. A continuación se describen los pasos para llevar a cabo una evaluación psicopedagíca: Secretaría de educación pública , S.f, pp 31-34.

\begin{abstract}
Aspectos principales de la identificación del alumnado con necesidades educativas especiales que ingresan a los planteles de Educación Básica regular: La identificación deberá realizarse de manera contextual e interdisciplinaria, considerando los siguientes momentos:
\end{abstract}

37.2.1. Detección Inicial: El proceso de detección inicial de los educandos con necesidades educativas especiales se desarrolla en tres etapas:

a) Evaluación inicial o diagnóstica del grupo. b) Evaluación más profunda de algunos educandos, con la finalidad de observarlos de manera más cercana y desarrollar estrategias que faciliten el aprendizaje y la participación de estos educandos. c) Solicitud de Evaluación Psicopedagógica.

37.2.2.

Evaluación

Psicopedagógica, para lo cual se deberá obtener la siguiente información, protegiendo en todo momento los datos sensibles que se recaben:

a) Datos Personales. b) Motivo de Evaluación. c) Apariencia Física. d) Conducta Durante la Evaluación. e) Antecedentes del Desarrollo: Embarazo, Antecedentes heredo-familiares, Desarrollo Motor, Desarrollo del Lenguaje, Historia Médica, Historia Escolar, Situación Familiar. f) Situación Actual: AREAS A EVALUAR - 1. Aspectos Generales: área intelectual, área de desarrollo motor, área comunicativolingüística, área de adaptación e inserción social, aspectos emocionales. 2. Evaluación de los aprendizajes. 3. Disposición al estudio, estrategias de estudio desarrolladas y aplicadas por el educando y motivación para 
aprender. 4. Información relacionada con el entorno del educando: contexto escolar y contexto socio-familiar. g) Interpretación de Resultados. h) Conclusiones y Recomendaciones. QUIENES PARTICIPAN 37.3. Trabajo Multidisciplinario: El (la) Director(a) de la institución educativa pública o particular con autorización, será el responsable de convocar a la primera reunión del equipo multidisciplinario de la escuela. En dicha reunión deben participar: Director(a), docente de grupo y de apoyo de USAER o CAPEP (en caso de que lo haya) y/o apoyos extraescolares. El propósito de dicha reunión será acordar los apoyos y las adecuaciones que se llevarán a cabo para el alumnado con Necesidades Educativas Especiales y/o Aptitudes Sobresalientes.

\section{AUTORIZACIÓN}

Realización de pruebas formales para la evaluación psicopedagógica: Se deberán tomar en cuenta los siguientes criterios: 38.1. Podrán realizarse en caso de que se cuente con personal capacitado para la aplicación de las mismas y si el educando lo requiere.

38.2. En caso de no contar con el personal capacitado para la realización de pruebas formales, el (la) Director(a) de la institución educativa pública o particular con autorización solicitará al personal de Educación Especial de la Entidad, la aplicación de dichas pruebas o asesoría para canalizar al educando con alguna institución o especialista.

38.3. Se informará previamente a la madre, al padre de familia o tutor(a) y al educando, sobre la aplicación de pruebas formales y se darán a conocer los objetivos de la realización de dichas pruebas.

\section{PARA DAR RESPUESTA -}

$41^{\text {a }}$ - Elaboración de la Propuesta Educativa Específica: La Propuesta Educativa Específica será elaborada por el (la) docente de grupo y de apoyo (en caso de que lo haya); participarán la familia del educando y el equipo multidisciplinario que se incluyó en el proceso de Evaluación Psicopedagógica. La Propuesta 
Educativa Específica, permanece con el (la) docente de grupo como una herramienta para su planeación cotidiana y será necesario que el personal del servicio de apoyo (en caso de que lo haya) cuente con una copia.

\section{La Propuesta Educativa} Específica para los educandos inscritos en planteles de Educación Básica regular, deberá contener los siguientes rubros: 41.1. Algunas consideraciones que es necesario especificar en la elaboración de la Propuesta Educativa Específica son las siguientes:

41.1.1. Partir del Informe de Evaluación Psicopedagógica y de la planeación del (de la) docente de grupo.

41.1.2. Definir los ajustes que requiere hacer la escuela en su organización, con el objetivo de brindarle los apoyos específicos requeridos y facilitar el aprendizaje y la participación de los educandos.

41.1.2.1. Definir las adecuaciones de acceso y los responsables para darle seguimiento, en caso de ser necesarias: en las instalaciones de la escuela y el aula y, si se precisa, especificar el tipo de ayudas personales o técnicas que necesita el educando en su proceso educativo, estableciendo las acciones requeridas para obtenerlas (dentro de la escuela y en el contexto socio-familiar).

41.1.2.2. Definir las adecuaciones curriculares que es necesario realizar en la metodología y en la evaluación, a partir de las habilidades y debilidades del educando, así como de los aprendizajes esperados.

41.1.3. Determinar la participación de la familia del educando:

41.1.3.1. Especificar los apoyos específicos que la familia brindará al educando, estableciendo compromisos.

41.1.3.2. En su caso, definir la información y la orientación que recibirá la familia por parte del equipo de apoyo y de la escuela.

Subsecretaría de Planeación y Evaluación de Políticas Educativas Dirección General de Acreditación, Incorporación y Revalidación 
41.1.4. En caso de ser necesario, determinar los apoyos específicos que se le brindarán al educando por parte del personal del servicio de apoyo:

41.1.4.1. En las actividades académicas.

41.1.4.2. En el aula de recursos.

41.1.4.3. Fuera de la escuela (apoyos complementarios o extracurriculares).

41.1.5. Acordar los compromisos que asumen los involucrados.

41.1.6. Establecer las fechas en las que se revisarán los avances del educando y se realizarán los ajustes necesarios.

41.2. La Propuesta Educativa Específica para los educandos en los Centros de Atención Múltiple contempla los siguientes rubros:

a) Apoyos profesionales. b) Apoyos materiales. c) Apoyos arquitectónicos. d) Apoyos curriculares. e) Apoyos generalizados. f) Apoyos permanentes.

Lo anterior describe la intervencón del docente en el aula, en el diagnostico de las Nesecidades Educativas Especiales. Para esto la descripción de los elementos de la evaluación psicopedagogica son fundamentales, en la detección y la puesta en marcha de acciones para que los alumnos alcancen los contenidos que marca el curriculum en el nivel en donde se encuentre. Con base a la información es importante resaltar que en este proceso de evaluación se ve implicado el equipo interdisciplinario: director, maestro aula regular y maestro de educación especial, quienes atienden la problemática implementado una popuesta curricular específica. Sin embargo, según lo establecido en la ley. Los padres deben ser informados y consultados para proceder con las pruebas y las adecuaciones correpondientes. Los progresos en el alumno serán monitoreados para registrar el avance, punto que valoramos como significativo en el proceso educativo de los alumnos con problemas de aprendizajes provocadas por un défict fisico, emocional o psicologico.

\section{Bibliografia}

BARRAZA, Macías, A., \& Cárdenas Aguila, T. Marco Conceptual y Experiencias de la Educación Especial en México. México: Instituto Universitario Anglo Español, 2014.

BECKY, L. Spivey, M. y.¿Qué es un maestro de educación especial? Super Duper ${ }^{\circledR}$ Handy Handouts!, 2. 2009. 
BOGGINO, N., \& de la Vega , E. (2006).

Diversidad, aprendizaje e integración en contextos escolares. Argentina: Homosapiens.

CARDONA, M. M. (2001). Adaptemos la escuela. Orientaciones ante la discapacidad motórica . España: Ajibe, 2001.

COMISIÓN DE POLÍTICA GUBERNAMENTAL EN MÉXICO DE DERECHOS HUMANOS. (s.f). Glosario de Términos sobre discapacidad. México: Secrtaría de Educación Pública. Diario Oficial de la Federación. En Ley Para la Protección de los Derechos de las Niñas, Niños y adolescentes (pág. 22). México: ANAYA EDITORES, S.A. 2000 .

DIARIO OFICIAL DE LA FEDERACIÓN. Ley para la atención y protección a personas con la condición del espectro autista. México. 2015. FRANZÉ, J. ¿Qué es polítca? España: Catarata. 2004.

GERDA, H. Las Nesecidades Educativas Especiales . Barcelona, España: Paidos. 2001.

GOBIERNO DE LA REPÚBLICA. Plan Nacional de Desarrollo. México. 20132018.

HEGARTY, s. Educaion de niños y jóvenes con discapacidad. Paris: Unesco. 1994.

MARES Miramontes, A., \& Ito Sugiyama, E. Integración educativa. Perspectiva desde los actores sociales encargados de realizarla . Revista Mexicana de Investigación Educativa, 29. (2005). MICHAEL W, A., \& James A, B. Escuelas Democr \{áticas . Madrid: Morata. 2000.

NEVILLE , J., \& Tim , S. Organización y

Función Directiva En Los Centros De Integración. España: La Muralla, S.A. 1995.

REGISTRO DE LA CÁMARA NACIONAL. Titulo Primero, Capitulo 1; De las garantías Individuales . En Constitución Política Educativa de los Estados Unidos Mexicanos (págs. 10-11). México: Anaya Editores, S.A. 2008.

SECRETARÍA DD EEDUCACIÓN PÚBLICA. Ley Federal de Educación . México. (1973).

SECRETARÍA DE EDUCACIÓN PÚBLICA . (S.f). Normas Específicas de Control Escolar Relativas a la Inscripción, Reinscripción, Acreditación, Promoción, Regularización y Certificación en la Educación Básica. México. 1973.

SECRETARÍA DE EDUCACIÓN PÚBLICA. Ley General de Educación. México: Diario Oficial de la Federación. 1993.

SECRETARÍA DE EDUCACIÓN PÚBLICA. Programa Nacional de Fotaecimiento de laEducación Especial y de la Integración Educativa. México: Secretaría de Educación Pública. 2001- 2006.

66 MUJICA, D.Y.R.; FELICIANO, T.M.; GUTIÉRREZ, M.Z.: Discusión Teórica Respecto A La Atención De Las Nee (Necesidades Educativas Especiales) En La Legislación Educativa Mexicana. 
SPIREY, B. ¿ Qué es un maestro de educación especial? Super Duper Publications, 166. 2009.

UNESCO. La inclusión educativa: el camino hacia el futuro . Ginebra: Unesco. 2008.

ZAMARA, A. M. (s.f). Políticas educativas en México. México. 2008. 